\title{
People who perceive themselves as active cannot identify the intensity recommended by the international physical activity guidelines
}

This article was published in the following Dove Press journal:

Open Access Journal of Sports Medicine

17 October 2014

Number of times this article has been viewed

\author{
Neal W Prokop' \\ Travis JR Hrubeniuk' \\ Martin Sénéchal ${ }^{2,3}$ \\ Danielle R Bouchard ${ }^{1,4}$ \\ 'Faculty of Kinesiology and \\ Recreation Management, University \\ of Manitoba, Winnipeg, Manitoba, \\ Canada; ${ }^{2}$ Manitoba Institute of Child \\ Health, Winnipeg, Manitoba, Canada; \\ ${ }^{3}$ Department of Pediatrics and Child \\ Health, Faculty of Medicine, University \\ of Manitoba,Winnipeg, Manitoba, \\ Canada; ${ }^{4} \mathrm{Health}$, Leisure, and Human \\ Performance Research Institute, \\ Winnipeg, Manitoba, Canada
}

Video abstract

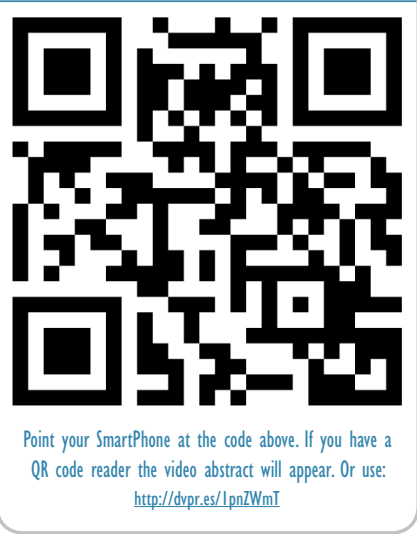

Correspondence: Danielle R Bouchard Faculty of Kinesiology and Recreation Management, University of Manitoba, 318 Max Bell Centre,Winnipeg, Manitoba R3T 2N2, Canada

$\mathrm{Tel}+\mathrm{I} 2044748627$

Fax + I 204 26| 4802

Email danielle_bouchard@umanitoba.ca
Background: Many national and international organizations recommend that adults achieve at least 150 minutes of aerobic physical activity (PA) weekly, at a minimum moderate intensity to optimize health benefits. It is unknown if people who consider themselves as active have the ability to identify what is considered moderate intensity.

Methods: Fifty-one participants who reported achieving a minimum 150 minutes per week at a minimum of moderate intensity PA were recruited through a local fitness facility. All participants underwent a single assessment involving questionnaires, clinical measures, and a treadmill test to measure the ability to perceive moderate intensity. Following the visit, participants' PA level was evaluated by heart rate monitor, while exercising, for 7 consecutive days.

Results: Eighty percent of participants overestimated moderate intensity on the treadmill test; they were at vigorous intensity compared to what is considered moderate. Only $11.8 \%$ of participants accurately identified moderate intensity; all of them were women $(P=0.03)$, had a high level of education $(P=0.04)$, and knew that moderate intensity was the minimum intensity recommended by health organizations $(P<0.01)$. Only $69.2 \%$ of participants reached the aerobic component of the International Physical Activity Guidelines with no significant advantage for those correctly identifying moderate intensity.

Conclusion: Most people who perceive themselves as active are exercising at vigorous intensity while believing they are at moderate intensity. In addition, in this active sample, one-third of the participants were not reaching the aerobic component of the International Physical Activity Guidelines.

Keywords: aerobic evaluation, health outcomes, physical exertion

\section{Introduction}

While health benefits are associated with any increment of physical activity (PA), ${ }^{1}$ greater benefits are observed when PA is performed at a minimum of moderate intensity in bouts of at least 10 minutes. $^{2}$ Most national and international PA guidelines (IPAG) recommend that adults achieve a minimum of 150 minutes of aerobic exercise at a minimum of moderate intensity, and two sessions of resistance training (RT) weekly, to optimize health benefits associated with active living. ${ }^{3-5}$

Of the three elements (ie, mode, duration, and intensity) prescribed by these IPAG, the intensity of aerobic exercise is definitely the most difficult to accurately monitor, as intensity monitoring tools (eg, heart rate [HR] monitor) might be unavailable or not understood. Self-reported PA measures are often unreliable and inconsistent because of confusion when defining PA. ${ }^{6}$ For example, more than half of adults self-reported themselves as reaching the IPAG, ${ }^{7,8}$ while objective measures of PA showed that 
only $15 \%$ of adults reach the aerobic component of these guidelines. ${ }^{9}$ Discrepancy between self-reported and objective measures of PA might be explained by the inability of identifying exercise intensity. As a consequence, individuals might not feel targeted when health professionals suggest that most individuals are not reaching IPAG. This is important from a public health perspective because despite many adults falling short of the IPAG, a large proportion of adults might believe they are optimizing their health through meeting the recommended guidelines.

In a previous study, it was shown that only $20 \%$ of inactive older adults could identify moderate intensity. ${ }^{10}$ It is also important to understand if active people can identify moderate intensity because they might be the segment of the population most receptive to optimizing their aerobic exercise sessions. If active people underestimate exercise intensity (perceive the task to be moderate, but it is actually at low intensity based on the individual physiological response), they might decrease the odds of meeting their goals and expectations, therefore negatively impacting their exercise adherence and PA levels. ${ }^{11}$ If individuals overestimate exercise intensity (perceive the task to be moderate, but it is actually at vigorous intensity based on the individual physiological response), they might decide to discontinue their current training when they become injured, sick, or older because of not being able to reach their perceived moderate intensity level, and thus assume they are not accumulating any additional health benefits.

The goals of this study were to examine if people who perceived themselves as active: 1) can identify moderate intensity; and 2) know and reach the IPAG. We also wanted to determine if unique characteristics were present among individuals who could identify moderate intensity, compared to individuals who could not identify the intensity. The results of this study have practical relevance for prescribing PA among a segment of the population that is potentially very receptive to interventions that optimize their exercise sessions. With an increasing recognition of the importance of PA to prevent chronic diseases, the ability for motivated people to accurately identify the minimum intensity necessary to optimize health benefits is an important consideration for long-term health promotion initiatives. We hypothesized that most active individuals, just like inactive individuals, would have difficulties identifying moderate intensity. We also hypothesized that the ability to identify moderate intensity might be indicative of which individuals reach the IPAG.

\section{Methods \\ Participants}

A total of 51 adults took part in this study. Recruitment was obtained by an email sent to all members of the University of Manitoba fitness facility and by poster advertisements within the facility. The facility has a running track, gym space, group classes, cardio equipment, and RT equipment. Inclusions criteria were: 1) age 18 years or above; 2) have medical clearance based on the PA Readiness Questionnaire; ${ }^{12}$ and 3) reported doing exercise for at least 150 minutes of moderate-to-vigorous intensity per week. Individuals with a pacemaker, using medications that affect HR, or that were educated in recognizing HR intensity (eg, cardiac rehabilitation program) were excluded from the current study because their exercise HR and perceived levels of exertion could be biased. The University of Manitoba Research Ethics Boards for humans approved the study protocol and written informed consent was obtained from each participant.

\section{Questionnaires}

Sociodemographic information (ie, age, sex, education level, and race/ethnicity) was self-reported by a questionnaire. Participants answered four questions regarding their knowledge of the IPAG. ${ }^{5}$ The first question was: "According to the IPAG, how much time do you think an adult of your age should do aerobic exercise per week to derive health benefits?" If participants reported a range, the mean was recorded. The second question was: "According to the IPAG, what is the minimum aerobic exercise intensity necessary to derive health benefits?" The possible answers were low, moderate, vigorous, or do not know. The third question was: "According to the IPAG, should an adult do RT to derive health benefits?" The fourth question was: "How many days per week should adults perform RT to derive health benefits?" The participant was expected to answer with a number, and if a range was reported, the mean was recorded.

\section{Clinical measures}

Resting HR was measured using a vital signs monitor (Model HEM711; Omron) following 5 minutes of inactivity during which the consent form was read and questionnaires were asked. Body weight was measured to the nearest $0.1 \mathrm{~kg}$ on a calibrated scale (SECA, Hamburg, Germany), and height was obtained with a standard stadiometer (Takei, Tokyo, Japan). Body mass index was calculated by dividing bodyweight $(\mathrm{kg})$ by height $(\mathrm{m})$ squared. All clinical measures 
were measured twice and the mean of both measurements was used for analyses. All clinical measures were collected as recommended by the Canadian Society of Exercise Physiology protocols. $^{13}$

\section{Identification of exercise intensity}

After a 3-minute warm-up to become accustomed with the treadmill, the IPAG was read to participants. They were then asked to self-adjust the speed and slope of the treadmill to what they considered as moderate-intensity exercise, or the minimum intensity necessary to derive health benefits. Participants were told they would need to maintain the pace for 5 minutes so HR could reach a steady state. During the test, speed, slope, and time were visible to participants; however, their HR was not. After 5 minutes, the participant's HR was calculated as a percentage of their HR reserve (HRR) as this is more associated with maximal capacity. ${ }^{14}$ HRR is calculated as maximal HR minus resting HR. Moderate intensity was defined as $40 \%-59 \%$ of a participant's HRR. ${ }^{15}$ In this text, overestimating refers to the instance in time where an individual is exercising at a higher intensity than they perceive. Underestimating refers to a scenario where an individual's perceived intensity is higher than what would be expected, based on their current HR while exercising.

\section{PA guidelines}

Following their assessment at our facility, participants were given a HR monitor, a PA log, and a pedometer to track physical activities for 7 consecutive days. No recommendation for type, duration, mode, or intensity of exercise was given to participants. In addition, no feedback was given regarding the results of their treadmill test, and their ability to accurately identify moderate intensity. During their week evaluation, participants were required to use a HR monitor (Polar Accurex Plus, Woodbury, NY, USA) that would be used to evaluate their activity duration and intensity levels for every exercise session. The HR was collected every 15 seconds and downloaded when the participant returned the equipment.
The PA log was used to determine the participant's specific mode of exercise that was performed (eg, aerobic versus RT). The HR monitor and PA log was also used to identify physical activities lasting longer than 10 minutes, and the pedometer (Stepscount, Deep River, Ontario, Canada) was worn by participants so that the total steps per day could be recorded in their PA log.

\section{Statistical analyses}

Data management and statistical analyses were performed using SPSS version 17.0 (SPSS Inc., Chicago, IL, USA). Normality of variables was tested by Shapiro-Wilk test. Results are reported as mean \pm standard deviation, for normally distributed variables. Median (25th-75th percentiles) was used to report descriptive variables not normally distributed, and N (\%) was used for categorical variables. Differences between participants who correctly identified moderate intensity or not were evaluated with Mann-Whitney tests and $t$-tests, for continuous variables, or chi-square tests and Fisher's exact test for categorical variables as appropriated.

\section{Results \\ Participant characteristics}

The general characteristics of our sample are presented in Table 1. The median age of all participants was 34 years and $43.1 \%$ of participants were male. Seventy-eight percent of the participants had a university or college education level and $76.5 \%$ of participants were Caucasian. The median body mass index of participants was $24.1 \mathrm{~kg} / \mathrm{m}^{2}$.

Only $11.8 \%$ of our participants accurately identified moderate intensity, based on their ability to accurately self-adjust the treadmill to a moderate intensity level during the treadmill test. When participants attempted to identify moderate intensity, the mean speed was $5.2 \mathrm{mph}$ and the mean slope was $1.0 \%$. Three-quarters of participants were running when they attempted to identify moderate intensity.

Table I General characteristics

\begin{tabular}{|c|c|c|c|c|}
\hline & Total sample $(n=5 I)$ & $\begin{array}{l}\text { Identified moderate } \\
\text { intensity }(n=6)\end{array}$ & $\begin{array}{l}\text { Did not identify moderate } \\
\text { intensity }(n=45)\end{array}$ & P-value \\
\hline Age & $34(23-55)$ & $54.5(22.5-62.0)$ & $34.0(22.8-52.5)$ & 0.57 \\
\hline Sex (men) & $23(43.1 \%)$ & $0(0 \%)$ & $23(48.9 \%)$ & 0.03 \\
\hline Education level (university) & $40(78.4 \%)$ & $5(83.3 \%)$ & $35(77.8 \%)$ & 0.04 \\
\hline Race/Ethnicity (Caucasian) & $39(76.5 \%)$ & $6(100.0 \%)$ & $33(73.3 \%)$ & 0.55 \\
\hline Body mass index $\left(\mathrm{kg} / \mathrm{m}^{2}\right)$ & $24.1(22.5-26.2)$ & 23.7 (19.5-27.2) & $24.1(22.5-26.0)$ & 0.60 \\
\hline
\end{tabular}

Note: Data are presented as median (25th-75th percentile) for variables or $n(\%)$ for categorical variables. 
Eighty percent of inaccurate participants overestimated moderate intensity and $8 \%$ underestimated moderate intensity. The participants accurately identifying moderate intensity were all women $(P=0.03)$. Participants with a university degree $(P=0.03)$ and Caucasians $(P=0.04)$ were also more likely to adjust the treadmill to moderate intensity.

\section{Perceptions of the PA guidelines}

The sample's perceptions of the IPAG are presented in Table 2. The perceived minimum time reported to maintain or improve health was 180 minutes. No difference was observed between the two groups when categorized by their ability to identify moderate intensity. Eighty-two percent of participants believed that moderate intensity was the minimum-needed aerobic-exercise intensity to maintain or improve health, while all participants recognized that RT is important to derive health benefits.

All participants $(n=6)$ who accurately identified moderate intensity during the submaximal treadmill test reported that moderate intensity was the minimum intensity level recommended by the IPAG compared to $80 \%$ of the group who did not accurately identify moderate intensity on the treadmill test $(P<0.01)$. In the group that did not identify moderate intensity, $17.8 \%$ believed that vigorous intensity was the minimum exercise level recommended by the IPAG, and this perception was different from the group that was able to identify moderate intensity $(P=0.03)$. No significant difference was observed between the two groups for the perceived importance of RT for health purposes and the number of days an individual should do this mode of exercise per week.

\section{PA level}

Participants' overall PA levels during their evaluation week are presented in Table 3. Sixty-nine percent of all participants reached the IPAG in terms of minutes of aerobic exercise at moderate-to-vigorous intensity. However, when considering RT, this proportion decreased to $34.6 \%$. There was no significant difference when comparing the accurate and non-accurate groups to determine a participant's likelihood of reaching the aerobic IPAG $(P=0.40)$. However, the group able to identify moderate intensity had a greater proportion of participants reaching the overall guidelines $(+17 \%)$ and accumulated more steps per day $(+14 \%)$, although these differences also did not reach significance. No difference was observed among groups for total time recorded or time spent at vigorous, moderate, and light intensity during the week evaluation.

\section{Discussion}

The main objective of this study was to examine if individuals who consider themselves active could identify the commonly prescribed exercise intensity (ie, moderate) to derive health benefits as prescribed by the IPAG. Another objective was to verify if the ability to identify exercise intensity was associated with the likelihood of individuals reaching the IPAG. The novel finding in our study is that only $11.8 \%$ of active adults, all of whom were women, were accurately able to identify the moderate PA intensity range. Eighty percent of inaccurate participants overestimated moderate intensity. The second important finding is that approximately one-third of our sample did not reach the aerobic components of the IPAG, despite believing that they regularly meet the aerobic IPAG during the week.

In this study, only six out of 51 participants $(11.7 \%)$ could accurately identify moderate intensity level while walking or running. This proportion is even lower from an inactive older sample $(20 \%)$ previously studied by our group. ${ }^{10}$ The biggest difference between the two samples is the fact that a large proportion of individuals who self-identify as active overestimated moderate intensity while older adults underestimated moderate intensity. ${ }^{10}$ A previous study has suggested that regular exercisers may underestimate physical exertion when using a self-perception measure normally based on the Borg scale, ${ }^{16}$ which helps exercisers evaluate their intensity

Table 2 Perceptions of international physical activity guidelines

\begin{tabular}{|c|c|c|c|c|}
\hline & Total sample $(n=5 I)$ & $\begin{array}{l}\text { Identified moderate } \\
\text { intensity }(n=6)\end{array}$ & $\begin{array}{l}\text { Did not identify moderate } \\
\text { intensity }(n=45)\end{array}$ & $P$-value \\
\hline Total exercise per week (min) & $180(150-300)$ & $150(90-185)$ & $180(150-300)$ & 0.39 \\
\hline \multicolumn{5}{|l|}{ Exercise intensity necessary } \\
\hline Light & I (I.9\%) & $0(0 \%)$ & I (2.2\%) & 0.89 \\
\hline Moderate & $42(82.3 \%)$ & $6(100 \%)$ & $36(80.0 \%)$ & $<0.01$ \\
\hline Vigorous & $8(15.7 \%)$ & $0(0 \%)$ & $8(17.8 \%)$ & 0.03 \\
\hline Is resistance training important (yes) & $51(100 \%)$ & $6(100 \%)$ & $46(100 \%)$ & 0.99 \\
\hline Resistance training (days/week) & $3(2-3)$ & $2(2-3)$ & $3(2-3)$ & 0.39 \\
\hline
\end{tabular}

Note: Data are presented as median (25th-75th percentile) for variables with an abnormal distribution, or $\mathrm{n}(\%)$ for categorical variables. 
Table 3 Physical activity level during 7-day evaluation

\begin{tabular}{|c|c|c|c|c|}
\hline & Total sample $(n=5 I)$ & $\begin{array}{l}\text { Identified moderate } \\
\text { intensity }(n=6)\end{array}$ & $\begin{array}{l}\text { Did not identify moderate } \\
\text { intensity }(n=45)\end{array}$ & $P$-value \\
\hline $\begin{array}{l}\text { Meeting the aerobic CPAG } \\
\text { (150 min/week at minimum intensity) }\end{array}$ & $36(69.2 \%)$ & $5(83.3 \%)$ & $31(68.9 \%)$ & 0.65 \\
\hline $\begin{array}{l}\text { Meeting the resistance CPAG } \\
\text { ( } 2 \text { days of resistance training) }\end{array}$ & $25(48.1 \%)$ & $4(66.7 \%)$ & $20(44.4 \%)$ & 0.40 \\
\hline Meeting the CPAG (yes) & 18 (34.6\%) & $3(50.0 \%)$ & 15 (33.3\%) & 0.65 \\
\hline Mean steps per day & $13,795(10,618-17,378)$ & $15,752(\mid 2,885-22,845)$ & $13,539(9,988-17,042)$ & 0.57 \\
\hline Total time recorded by the HR monitor & $421(316-544)$ & $423(330-610)$ & $421(313-548)$ & 0.68 \\
\hline Vigorous intensity (min) & $93(54-146)$ & $101(40-168)$ & $92(54-142)$ & 0.89 \\
\hline Moderate intensity (min) & $89(43-190)$ & $83(19-163)$ & $89(44-193)$ & 0.47 \\
\hline Light intensity (min) & $164(83-300)$ & $173(132-488)$ & $164(80-296)$ & 0.41 \\
\hline
\end{tabular}

Note: Data are presented as median (25th-75th percentile) for variables with an abnormal distribution or $n$ (\%) for categorical variables.

Abbreviations: CPAG, Canadian Physical Activity Guidelines; HR, heart rate.

perception on a scale between 6 and 20 or 0 and 10 . However, the sample recruited for that study was older ( $38 \%$ aged 50 years and over versus $26 \%$ in the present study), heavier ( $20.7 \%$ obese versus $3.9 \%$ ), and participants were eligible simply by being a member of a fitness facility despite their PA level, while we only recruited members that reported achieving a minimum of 150 minutes of moderate aerobic exercise weekly.

At first sight, it might not seem problematic that people are overestimating their PA intensity; however, there are advantages and disadvantages to inaccurately identifying exercise intensity. The obvious advantage of exercising at a greater intensity is that it provides more health benefits. ${ }^{17} \mathrm{Also}$, some argue that vigorous intensity with short durations can reach similar or superior health benefits, and by the same token, reduce the total time that one needs to exercise weekly. ${ }^{18}$ Since lack of time is a main limitation to being active, ${ }^{19}$ the ability to exercise at a vigorous intensity would help further improve the health of individuals. Conversely, there can also be disadvantages to overestimating moderate exercise intensity. First, this could potentially lead to overtraining or an increased risk of injury. Second, when these active individuals get old, sick, or face barriers, the overestimation of moderate intensity might cause individuals to associate moderate intensity with light exercise ${ }^{19,20}$ and provoke them into thinking they are not accumulating worthwhile health benefits. In other words, individuals who do not think they will be able to achieve the minimum intensity during a session might avoid the entire exercise session altogether. Although accurate perceptions does not guarantee adherence to PA, individuals should still strive for awareness and knowledge regarding the PA guidelines and their PA intensity levels.

Our results show that highly educated women are more likely to identify the minimum intensity recommended by several health organizations. In fact, $83.3 \%$ of women who correctly identified moderate intensity had a university degree. It is known that high education level, university in this case, is associated with broader knowledge about health-related topics, ${ }^{21}$ and women are more likely be aware of strategies to maintain or improve health. ${ }^{22}$ For these reasons, it was not a surprise to see women and education level associated with the ability to identify moderate intensity.

An interesting finding was that most of our participants exceeded the recommended 10,000 steps per day, ${ }^{23}$ yet almost two-thirds (65.4\%) of our assumed active sample did not reach the overall IPAG (aerobic plus RT). This was surprising since all participants reported that RT was important, and the inclusion criteria required individuals to self-report doing more than 150 minutes of moderate intensity exercise. The HR monitors and 7-day PA logs suggest that an association may exist between people who are able to identify moderate intensity and the likelihood to reach the IPAG. Due to the small sample size, further investigation is needed to identify if the ability to identify exercise intensity helps individuals meet the IPAG. In this study, the difference was $14.4 \%$ for aerobic guidelines, $22.3 \%$ for RT guidelines, and $14.1 \%$ for steps per day, in favor of the group able to identify moderate intensity.

Another component of the IPAG is related to RT. The IPAG suggest a minimum of two times per week of RT, and the vast majority of participants reported that two to three sessions should be done. In this sample, participants had unlimited access to RT equipment, but only $48.1 \%$ of them choose to use the equipment two times or more during the week evaluation. Since RT has been shown to have a significant impact on health, ${ }^{24,25}$ future interventions could target the large proportion of individuals who are already active members of fitness facilities but decide to avoid this mode of exercise training. 
Our study is strengthened by the use of an objective intensity measure (ie, HR monitor) for 7 consecutive days and a well-characterized sample. However, some limitations need to be highlighted. First, people self-identified themselves as active during recruitment, which may have not been indicative of their actual lifestyle. However, the mean steps per day was 13,795 and $70 \%$ of them were doing a minimum of 150 minutes of PA at moderate-to-vigorous intensity. Because of the low number of participants in the study, and the small proportion of individuals who correctly identified moderate intensity, it is difficult to fully explain and investigate their characteristics with certainty. A sample of inactive people to compare our studied group to would have strengthened our results. However, it can be compared with a sedentary sample previously studied by other groups. We relied on a self-reported exercise type to determine if they performed RT during the week evaluation. It is possible that participants were more likely to overestimate their exercise levels because of desirability to show the research assistant that moderate intensity was easy. While it is believed that participants wore their HR monitors for all physical activities during their week evaluation, it is possible the monitor was ignored or forgotten by participants during some of their activities. In this study, the main motive to exercise (eg, health, performance) was not captured. This information could help identify if overestimating exercise intensity is really a concern to avoid overtraining or exhaustion. Finally, it is possible that the 7-day evaluation period is not reflective of the participants' regular exercise regimen.

\section{Conclusion}

In summary, our results suggest that individuals who selfidentify as active are not able to identify what is considered moderate intensity, and are mostly overestimating exercise intensity. Simple and affordable strategies to identify physical intensity should be developed to help adults, even those who consider themselves active, identify what is needed to reach these guidelines and derive additional health benefits. Such findings have practical relevance for a group of people that is potentially very receptive to an exercise program focusing on both the duration and intensity of the performed PA.

\section{Acknowledgment}

This study was funded by the University of Manitoba Faculty of Kinesiology and Recreation Management Dean's grant.

\section{Disclosure}

The authors report no conflicts of interest in this work.

\section{References}

1. Macfarlane DJ, Taylor LH, Cuddihy TF. Very short intermittent vs continuous bouts of activity in sedentary adults. Prev Med. 2006;43(4): 332-336.

2. Powell KE, Paluch AE, Blair SN. Physical activity for health: What kind? How much? How intense? On top of what? Annu Rev Public Health. 2011;32:349-365.

3. WHO global recommendation on physical activity for health [webpage on the Internet]. Geneva: World Health Organization; 2010. Available from: http://www.who.int/dietphysicalactivity/factsheet_recommendations/ en/. Accessed September 24, 2013.

4. Physical Activity Guidelines for Americans [webpage on the Internet]. Atlanta: Centers for Disease Control and Prevention; 2008. Available from: http://www.cdc.gov/physicalactivity/everyone/guidelines/adults. html. Accessed September 24, 2013.

5. gov.ik [homepage on the Internet]. UK Physical Activity Guidelines. Department of Health; 2011. Available from: http://www.gov.uk/ government/publications/uk-physical-activity-guidelines. Accessed February 12, 2014.

6. Katzmarzyk PT, Tremblay MS. Limitations of Canada's physical activity data: implications for monitoring trends. Can J Public Health. 2007;98 Supp1 2:S185-S194.

7. Bryan SN, Katzmarzyk PT. Are Canadians meeting the guidelines for moderate and vigorous leisure-time physical activity? Appl Physiol Nutr Metab. 2009;34(4):707-715.

8. Ready AE, Butcher JE, Dear JB, et al. Canada's physical activity guide recommendations are a low benchmark for Manitoba adults. Appl Physiol Nutr Metab. 2009;34(2):172-181.

9. Colley RC, Garriguet D, Janssen I, Craig CL, Clarke J, Tremblay MS. Physical activity of Canadian adults: accelerometer results from the 2007 to 2009 Canadian Health Measures Survey. Health Rep. 2011;22(1):7-14.

10. Bouchard DR, Langlois MF, Boivert-Vigneault K, Farand P, Paulin M, Baillargeon JP. Pilot study: can older inactive adults learn how to reach the required intensity of physical activity guidelines? Clin Interv Aging. 2013;8:501-508.

11. Annesi JJ. Goal-setting protocol in adherence to exercise by Italian adults. Percept Mot Skills. 2002;94(2):453-458.

12. Jamnik VK, Gledhill N, Shephard RJ. Revised clearance for participation in physical activity: greater screening responsibility for qualified university-educated fitness professionals. Appl Physiol Nutr Metab. 2007;32(6):1191-1197.

13. Canadian Society of Exercise Physiology. The Canadian Physical Activity, Fitness and Lifestyle Approach (CPAFLA): CSEP-Health and Fitness Program's Health-Related Appraisal and Counselling Strategy. 3rd ed. 2009.

14. Swain DP, Leutholtz BC. Heart rate reserve is equivalent to $\% \mathrm{VO} 2$ reserve, not to \%VO2max. Med Sci Sports Exerc. 1997;29(3):410-414.

15. Warburton DE, Nicol CW, Bredin SS. Prescribing exercise as preventive therapy. CMAJ . 2006;174(7):961-974.

16. Skatrud-Mickelson M, Benson J, Hannon JC, Askew EW. A comparison of subjective and objective measures of physical exertion. J Sports Sci. 2011;29(15):1635-1644.

17. Swain DP, Franklin BA. Comparison of cardioprotective benefits of vigorous versus moderate intensity aerobic exercise. Am J Cardiol. 2006;97(1):141-147.

18. Gibala MJ, Little JP, Macdonald MJ, Hawley JA. Physiological adaptations to low-volume, high-intensity interval training in health and disease. J Physiol. 2012;590(Pt 5):1077-1084.

19. Cerin E, Leslie E, Sugiyama T, Owen N. Perceived barriers to leisuretime physical activity in adults: an ecological perspective. $J$ Phys Act Health. 2010;7(4):451-459. 
20. Stutts WC. Physical activity determinants in adults. Perceived benefits, barriers, and self efficacy. AAOHN J. 2002;50(11):499-507.

21. Baum S, Ma J, Payea K. The Benefits of Higher Education for Individuals and Society. Trends in Higher Education Series. College Board Advocacy and Policy Center. 2010:56.

22. Courtenay WH, McCreary DR, Merighi JR. Gender and ethnic differences in health beliefs and behaviors. J Health Psychol. 2002;7(3): 219-231.
23. Choi BC, Pak AW, Choi JC, Choi EC. Daily step goal of 10,000 steps: a literature review. Clin Invest Med. 2007;30(3):E146-E151.

24. Artero EG, Lee DC, Lavie CJ, et al. Effects of muscular strength on cardiovascular risk factors and prognosis. J Cardiopulm Rehabil Prev. 2012;32(6):351-358.

25. Artero EG, Lee DC, Ruiz JR, et al. A prospective study of muscular strength and all-cause mortality in men with hypertension. J Am Coll Cardiol. 2011;57(18):1831-1837.

\section{Publish your work in this journal}

Open Access Journal of Sports Medicine is an international, peer-reviewed, open access journal publishing original research, reports, reviews and commentaries on all areas of sports medicine. The manuscript management system is completely online and includes a very quick and fair peer-review system.
Visit http://www.dovepress.com/testimonials.php to read real quotes from published authors. 